\title{
InTransBo: An Integrative Transcript Library to Enable Genome-Free Systematic Exploration of Bougainvillea
}

\section{Qi Luo}

The R\&D Group of the General Engineer Office, The Road Development Center of Xiamen, Xiamen, 361000

\section{Ziliang Chen}

The Wanyin Environmental Technology Limited Company, Xiamen, 361000

\section{Tingting Xu}

School of Life Sciences, Xiamen University, Xiamen, 361102

\section{Dangzheng Huang}

The R\&D Group of the General Engineer Office, The Road Development Center of Xiamen, Xiamen, 361000

\section{Haitao Hou}

The R\&D Group of the General Engineer Office, The Road Development Center of Xiamen, Xiamen, 361000

\section{Chenjie Hong}

The R\&D Group of the General Engineer Office, The Road Development Center of Xiamen, Xiamen, 361000

\section{Fulin Zhan}

The Wanyin Environmental Technology Limited Company, Xiamen, 361000

\section{Hangqi Guo}

The Wanyin Environmental Technology Limited Company, Xiamen, 361000

\section{Zhe Lin}

School of Life Sciences, Xiamen University, Xiamen, 361102

\section{Xiaoling Guo}

College of the Environment \& Ecology, Xiamen University, Xiamen, 361102

\section{Liang Chen}

School of Life Sciences, Xiamen University, Xiamen, 361102

\section{Zhi-Liang Ji ( $\square$ appo@xmu.edu.cn )}

School of Life Sciences, Xiamen University, Xiamen, 361102

\section{Research Article}


Keywords: Bougainvillea, InTransBo, RNA library, Transcriptome

Posted Date: December 31st, 2020

DOI: https://doi.org/10.21203/rs.3.rs-134688/v1

License: (c) (1) This work is licensed under a Creative Commons Attribution 4.0 International License. Read Full License 


\section{Abstract}

Members of the genus Bougainvillea are rich sources of natural dyes, pigments, and traditional medicines. They are also commonly used as ornamentals in roadside landscape construction. However, the horticultural development of Bougainvillea flowers with extended growth periods and coloration is not always feasible. One reason is limited molecular knowledge and no genomic information for Bougainvillea. Here, we compiled an expressed transcript sequence library for Bougainvillea by integrating 20 Illumina-sequencing RNA transcriptomes. The library consisted of 97,623 distinct transcripts. Of these, 47,006 were protein-coding, 31,109 were IncRNA, and 19,508 were unannotated. We also confirmed that the library is an alternative genomic reference for accurate transcriptome assembly and its performance was substantially better than that of the de novo method. We also curated the Integrative Transcript Library database for Bougainvillea known as InTransBo (http://www.bioadd.org/InTransBo/index.jsp). To the best of our knowledge, the present study is the first large scale genomic resource for Bougainvillea. Overall, the library helps fill the genomic gap and elucidate the transcriptional nature of Bougainvillea. It may also advance progress in the precise regulation of flowering in horticulture. The same strategy can be readily applied toward the systematic exploration of other plant species lacking complete genomic information.

\section{Introduction}

Bougainvillea sp. are native to the Amazonian rainforests of South America but are globally distributed. They are used mainly as ornamental and landscaping plants (Tsai, Su, Liao, \& Hung, 2005). Bougainvillea sp. belongs to the family Nyctaginaceae and comprises $\sim 18$ species (B. berberidifolia, $B$. buttiana, $B$. campanulata, B. glabra, B. herzogiana, B. infesta, B. lehmanniana, B. lehmannii, B. malmeana, $B$. modesta, B. pachyphylla, B. peruviana, B. pomacea, B. precox, B. spectabilis, B. spinosa, B. stipitata, and B. trollii) (Abarca-Vargas \& Petricevich, 2018). To date, over 100 cultivars and three major hybrids have been recognized (Abarca-Vargas \& Petricevich, 2018; Tsai et al., 2005). However, only four species ( $B$. buttiana, B. glabra, B. spectabilis, and B. peruviana) have been commercially exploited (Choudhary, Kapoor, \& Lodha, 2008).

Bougainvillea sp. have been extensively investigated since 1970 as potential sources of traditional medicine (Hernandez-Martinez, Estevez, Vargas, Quintanilla, \& Rodriguez, 2011; Heuer et al., 1994). Aqueous Bougainvillea extracts and decoctions are used for fertility control by the tribal people of several countries (Ghogar \& Jiraungkoorskul, 2017). Bougainvillea may also have anticancer, antidiabetic, antihepatotoxic, anti-inflammatory, antihyperlipidemic, antimicrobial, antioxidant, and anti-ulcer properties (Arteaga Figueroa, Barbosa Navarro, Patino Vera, \& Petricevich, 2015). The alkaloids, essential oils, flavonoids, glycosides, oxalates, phenolics, phlobotannins, quinones, saponins, tannins, and terpenoids in Bougainvillea sp. might account for their putative medicinal properties (Saleem et al., 2019). Bougainvinones, pinitol, quercetagetin, quercetin, and terpinolene may also contribute to the therapeutic efficacy of Bougainvillea (Ghogar \& Jiraungkoorskul, 2017; Hernandez-Martinez et al., 2011). 
Analyses of the metabolites (Sangthong, Suksabye, \& Thiravetyan, 2016), natural dyes and pigments (Sangthong et al., 2016), medicinal uses, and species diversity of Bougainvillea have been conducted. In contrast, there have been few molecular studies of this genus. No genome of Bougainvillea has ever been sequenced up-to-date; and it won't be done in the coming few years due to technical and economic difficulties. Limited omics research has been performed to elucidate the molecular basis of the aforementioned properties of Bougainvillea especially at the systematic level. As no genome has been clarified for Bougainvillea, current molecular research on this plant is often compared against or referred to Arabidopsis thaliana. Consequently, its gene behavior is uncertain, ambiguous, or even misunderstood as there are genomic gaps between organisms. Therefore, an alternative genomic resource is required that can complement or fill the no-genome gap. Here, our objectives were to use multiple Illumina RNA sequencing (RNA-seq) transcriptomes determined for various Bougainvillea tissues and generate a sequence library consisting of all expressed transcripts. This library could serve as an alternative genomic reference for the molecular exploration of Bougainvillea and will be presented as an online interactive database.

\section{Materials And Methods}

\section{Sample Collection}

Tissue samples of pot-grown Bougainvillea glabra L. Choisy Tonganhong) in its normal flowering period were collected in October 2018 at Xiamen City, P.R. China. Samples comprised thorns, flower thorns, small buds, bracteoles, leaf sprouts, flower sprouts, lobules, stems under buds, stems under bracteoles, and flowers. Two biological replicates per tissue type were used (Table 1). The tissues were excised either from different parts of the same plant or from different plants, washed with distilled water, and briefly airdried in a clean environment. The tissues were mixed, randomly divided into two replicates, packed in silver paper, frozen in liquid nitrogen, and stored in the School of Life and Science, Xiamen University, Chen's lab. 
Table 1

Illumina RNA-sequencing datasets used in this study.

\begin{tabular}{|c|c|c|c|c|c|c|c|}
\hline Tissue & Raw reads & $\begin{array}{l}\text { Clean } \\
\text { reads }\end{array}$ & $\begin{array}{l}\text { Raw } \\
\text { base } \\
\text { (G) }\end{array}$ & $\begin{array}{l}\text { Clean } \\
\text { base } \\
\text { (G) }\end{array}$ & $\begin{array}{l}\text { Q20 } \\
(\%)\end{array}$ & $\begin{array}{l}\text { Q30 } \\
(\%)\end{array}$ & $\begin{array}{l}\text { GC } \\
\text { content } \\
(\%)\end{array}$ \\
\hline thorn & $33,613,973$ & $31,358,904$ & 10.08 & 9.41 & 97.68 & 93.60 & 43.13 \\
\hline thorn replicate 1 & $28,729,693$ & $27,644,873$ & 8.62 & 8.29 & 97.47 & 93.09 & 43.16 \\
\hline flower thorn & $26,343,968$ & $25,698,944$ & 7.90 & 7.71 & 96.26 & 90.50 & 42.95 \\
\hline $\begin{array}{l}\text { flower thorn replicate } \\
1\end{array}$ & $34,229,176$ & $32,719,720$ & 10.27 & 9.82 & 97.72 & 93.73 & 42.91 \\
\hline small bud & $31,318,836$ & $23,553,700$ & 9.39 & 7,07 & 97.47 & 93.24 & 42.84 \\
\hline small bud replicate 1 & $29,759,552$ & $21,379,446$ & 9.93 & 8.63 & 93.56 & 85.86 & 42.55 \\
\hline bracteole & $31,247,574$ & $30,110,664$ & 9.37 & 9.03 & 97.78 & 93.90 & 42.92 \\
\hline bracteole replicate 1 & $32,190,609$ & $30,967,796$ & 9.66 & 9.29 & 97.66 & 93.62 & 42.38 \\
\hline leaf sprout & $30,698,035$ & $30,294,725$ & 9.21 & 9.09 & 97.71 & 93.72 & 42.41 \\
\hline $\begin{array}{l}\text { leaf sprout replicate } \\
1\end{array}$ & $29,239,014$ & $28,026,332$ & 8.77 & 8.41 & 97.66 & 93.62 & 42.80 \\
\hline flower sprout & $32,856,663$ & $31,772,572$ & 9.86 & 9.53 & 97.35 & 92.94 & 42.85 \\
\hline $\begin{array}{l}\text { flower sprout } \\
\text { replicate } 1\end{array}$ & $33,433,464$ & $32,960,179$ & 10.03 & 9.89 & 97.70 & 93.65 & 42.87 \\
\hline lobule & $30,645,057$ & $30,328,864$ & 9.19 & 9.10 & 96.39 & 90.81 & 42.80 \\
\hline lobular replicate 1 & $29,613,243$ & $29,302,625$ & 8.88 & 8.79 & 97.71 & 93.74 & 42.50 \\
\hline stem under bud & $30,698,035$ & $30,294,725$ & 9.21 & 9.09 & 97.71 & 93.72 & 42.41 \\
\hline $\begin{array}{l}\text { stem under bud } \\
\text { replicate } 1\end{array}$ & $33,460,411$ & $31,701,317$ & 10.04 & 9.51 & 98.04 & 94.24 & 42.99 \\
\hline stem under bracteole & $30,982,149$ & $29,649,261$ & 9.29 & 8.89 & 96.03 & 90.09 & 42.89 \\
\hline $\begin{array}{l}\text { stem under bracteole } \\
\text { replicate } 1\end{array}$ & $33,115,209$ & $31,568,381$ & 9.93 & 9.47 & 96.42 & 90.82 & 42.69 \\
\hline flower & $32,141,549$ & $30,546,299$ & 9.64 & 9.16 & 97.59 & 93.45 & 42.90 \\
\hline flower replicate 1 & $31,700,976$ & $30,410,918$ & 9.51 & 9.12 & 97.36 & 92.93 & 42.42 \\
\hline
\end{tabular}

\section{RNA Library Construction and Deep Sequencing}

The sample mixtures were lysed with $1 \mathrm{~mL}$ TRIzol reagent (Invitrogen, Carlsbad, CA, USA). Total RNA was prepared according to the manufacturer's instructions. RNA purity was evaluated with a NanoPhotometer® spectrophotometer (Implen USA, Westlake Village, CA, USA). The RNA concentrations 
were measured with a Qubit ${ }^{\circledR}$ RNA assay kit and a Qubit® 2.0 fluorometer (Life Technologies, Waltham, MA, USA). RNA integrity was evaluated with the RNA Nano 6000 assay kit in an Agilent Bioanalyzer 2100 system (Agilent Technologies, Santa Clara, CA, USA). RNA samples passing the quality control test were stored at $-20{ }^{\circ} \mathrm{C}$ until later use.

The RNA library was constructed by Novogene Co. Ltd. (Beijing, China) following the standard operation procedure. Three micrograms RNA per sample was used as the input material for library preparation. Polyadenylated (poly(A)) RNA was purified from total RNA with poly T oligo-attached magnetic beads. RNA sequencing was performed in an Illumina HiSeq 4000 system (Illumina, San Diego, CA, USA) using the 125-bp, strand-specific, paired-end mode.

\section{RNA-Seq Data Preprocessing}

Before proceeding to the transcriptome assembly, the RNA-seq raw data were filtered with Trimmomatic (Bolger, Lohse, \& Usadel, 2014) to exclude low-quality reads and those with adaptors or $\mathrm{N}$ content $>10 \%$. The proportion of bases with $s Q \leq 5$ occurred in $>50 \%$ of all reads.

\section{Construction of the Integrative Transcript Library}

An integrative transcript library was constructed with TransIntegrator (Qin et al., 2020). TransIntegrator required four continuous steps for transcript library construction. Here, the computational workflow was specified as follows: (1) 20 RNA-seq datasets were de novo assembled separately with Trinity software (version 1.0) by contig length $>200 \mathrm{bp}, \mathrm{K}$-mer $>5$, and max reads of per graph $>200 \mathrm{bp}$ (Haas et al., 2013). (2) All expressed transcripts were clustered with CD-HIT-EST (version 4.5.4) (Fu, Niu, Zhu, Wu, \& Li, 2012) using a sequence identity threshold $-c>90 \%$ and an alignment coverage $-a S>80 \%$. The clustered sequences were identified, the longest sequences were selected as representatives, and the shorter sequences were discarded. (3) The representative transcripts were bridged with CAP3 (version 12/21/07, default parameters) to form longer sequences (Huang \& Madan, 1999) when two sequences had $>40 \mathrm{bp}$ overlap ( $>90 \%$ sequence identity). (4) The library was refined by removing sequences $<300 \mathrm{bp}$, and the mature Bougainvillea transcript library was obtained.

A conventional procedure was followed to annotate the integrative transcript library. The coding transcripts were identified with TransDecoder v3.0.0 (parameter: default) (Haas et al., 2013) by referring to the SwissProt and Pfam databases. The rRNA was annotated with RNAmmer (version 1.0.0, default parameters) (Lagesen et al., 2007). The tRNA was annotated with tRNAscan-SE (version 2.1.3, default parameters) (Lowe \& Eddy, 1997). The ncRNAs and miRNA precursors were identified by using the BLAST tool (version 1.3.1, e-value:1e-5, identify:90\%) against the NONCODE database (Xie et al., 2014) and miRBase (Kozomara \& Griffiths-Jones, 2013). All annotations were then integrated and those with low confidence (subject to the default threshold of corresponding annotation tools) were discarded.

\section{Library-Based Transcriptome Assembly and Performance Comparison}


To make a genomic reference for the transcriptome assembly, the library was annotated and preformatted as a gff file with Annoscript (version 1.1.3, default parameters) (Musacchia, Basu, Petrosino, Salvemini, \& Sanges, 2015). For library-based transcriptome assembly, we used HISAT software (version 1.3.1, min-intronlen:200 bp, pen-noncansplice:20, no-spliced-alignment) and quantified by HTSeq (version 3.2, default parameters) (Anders, Pyl, \& Huber, 2015). For de novo transcriptome assembly, we used Trinity software V1.0 as described above.

The transcriptome assemblies of the library-based and de novo methods were compared by using two external Bougainvillea RNA-seq datasets derived from public resources (SRA: SRR10097433 and SRR2912684). The comparison implemented several statistical parameters such as the reads mapping ratio, $\mathrm{N}_{50}$, and the unique gene (unigene) number (Moreton, Izquierdo, \& Emes, 2015). Of them, $\mathrm{N}_{50}$ is a

common indicator to evaluate genome assembly quality, the larger the better. In this study, $\mathrm{N}_{50}$ was used to indicate general transcript assembly quality. $A$ large $N_{50}$ suggested more long transcripts were assembled. The completeness and fragmentation ratio of the transcripts were evaluated with BUSCO (version 2.1.3, default parameters) (Simao, Waterhouse, loannidis, Kriventseva, \& Zdobnov, 2015). The potential bridge and assembly score were determined with TransRate (version 1.3, default parameters) (Smith-Unna, Boursnell, Patro, Hibberd, \& Kelly, 2016).

\section{Database Construction}

For user convenience, the transcript library was presented as an online interactive database called InTransBo, which was constructed on the Linux-Apache-JSP platform. MySQL software was used to manage data storage, access, and maintenance. Efficient and friendly user interfaces were designed with JavaScript for interactive transcript search and retrieval.

\section{Results And Discussion}

\section{Construction of the Integrative Transcript Library for theBougainvillea}

After quality control, the 20 RNA-seq datasets generated $\sim 590.30$ million clean reads and 172.23 gigabase pairs (Gbp). Detailed information on these datasets was summarized in Table 1. Integration of these datasets yielded a Bougainvillea transcript library comprising 97,623 non-redundant sequences. Transcript integrity was supported by multiple mapping reads.

The average library transcript length was $724.11 \mathrm{bp}$. The average library GC content was 40.12. About $77.03 \%$ of the transcripts were in the range of $300-885 \mathrm{bp}$ long which agreed with a previous plant gene length estimation of 183-2,000 bp (Cheng et al., 2017). Hence, most of the library transcripts were correctly assembled. Of the 97,623 sequences, $~ 48.15 \%(47,006)$ were annotated as protein-coding transcripts, 31.87\% $(31,109)$ were annotated as potential long non-coding RNAs (IncRNAs) and other non-coding RNAs (ncRNAs), and the remaining 19.98\% (19,508) were not annotated (Fig. 1a). 
We performed a gene conservation analysis by mapping the expressed Bougainvillea transcripts against the Arabidopsis thaliana genome. We found that 44,344 transcripts had homologs in the Arabidopsis thaliana genome, 24,870 transcripts mapped to intergenic regions, and 28,409 were unmapped (Fig. 1). The latter were either annotated Bougainvillea-specific or wrongly assembled transcripts. We selected ten conserved plant genes to verify sequence completeness. To this end, we compared them with their homologs in Arabidopsis thaliana (Fig. 1b). Out of the ten Bougainvillea genes, seven had sequence identity and coverage $>90 \%$ of their corresponding homologs in Arabidopsis thaliana. The remaining three genes failed either criterion. Thus, we confirmed the sequence completeness of the transcript library.

\section{Integrative Transcript Library Database for theBougainvillea}

The InTransBo database is freely accessible at http://www.bio-add.org/InTransBo/ or at its mirror site http://bioinf.xmu.edu.cn/InTransBo/. InTransBo uses keyword search and sequence BLAST to retrieve data interactively. The keyword search function allows both accurate and fuzzy transcript search via the input of complete or partial gene symbols, gene names, protein names, and abbreviated protein names (Fig. 2a). The search engine feeds back the hit terms in alphabet order along with the gene symbols, protein names, transcript lengths, and transcript ID. Clicking on the transcript ID redirects to the transcript information page containing various data listed in order including transcript annotations, sequence information, and transcript expression profiles (Fig. 3).

For newly identified sequences and unnamed sequences, InTransBo enables data access via an alternative BLAST method. The database supports BLASTn and tBLASTn to identify nucleic acid and protein sequences, respectively. The input may either be the typing sequence in text form or an uploaded file in FASTA format (Fig. 2b). The embedded BLAST engine responds to all hit sequences meeting the default expectation threshold of E value $=1 \mathrm{e}-10$ and sorted by hit score (Fig. 2b). For each hit, alignment details may be obtained using the "Alignment" hyperlink. Detailed information of the hit transcript may be acquired via the transcript ID hyperlink. The transcript library is free for downloading; however, user registration is required. The library is annotated and formatted as a gff file such that it may be used as an alternative reference to the genome for transcriptome assembly and other molecular applications.

\section{Library-based Transcriptome Assembly}

The library was used to demonstrate the reference-based assembly of 20 RNA-seq transcriptomes according to a typical genome-based method. The gene expression profiles in different tissues are accessed in the InTransBo database by specifying gene symbols. We compared transcriptome assembly performance between the library-based and de novo Trinity methods. The comparison was tested on two external Bougainvillea RNA-seq datasets (Table 1) determined by different research groups under various experimental conditions and using diverse sequencing qualities. Assembly performance was evaluated based on the read mapping ratio, number of unique genes and bridges, sequence completeness, fragmentation ratio, and estimated assembly score. The results are illustrated in Fig. 4. The library-based method substantially outperformed the de novo Trinity method. The former had superior N50, lower 
fragmentation ratio, and higher assembly score. The Trinity-assembled transcriptomes had relatively higher read mapping ratios and unigene numbers. However, the Trinity method also considered numerous fragmental sequences that could not be correctly assembled.

Bougainvillea sp. require systematic transcriptome research but lack genome support. Whole-genome sequencing is not a viable option to-date as it is costly and technically impracticable. Here, we used the computational method TransIntegrator to construct an integrative transcript library for Bougainvillea based on 20 heterogeneous RNA-seq datasets. We curated the InTransBo database on the transcript library for interactive data retrieval. By using this library, we demonstrated reference-based transcriptome assembly for the 20 RNA-seq transcriptomes. To the best of our knowledge, this study is the first to investigate Bougainvillea at the molecular level with no genome, and the InTransBo database could be the first genomic sequence source for Bougainvillea. A subsequent analysis revealed that the librarybased method outperformed the de novo Trinity method in terms of transcriptome assembly. Therefore, the library may serve as a reliable alternative reference that replaces the genome and enables the molecular exploration of Bougainvillea. The present study showed that the InTransBo database mitigates the genome constraint on the systematic investigation of Bougainvillea. We believe that it also helps elucidate the molecular biology of Bougainvillea and facilitates precise flower regulation in horticultural practices. The same strategy could be readily applied toward the systematic exploration of other plant species lacking adequate genomic data.

\section{Declarations}

\section{ACKNOWLEDGMENTS}

Financial support from the Transportation Technology Project of Fujian Province (No. 2018Y002) are gratefully acknowledged.

\section{AVAILABILITY OF DATA AND MATERIALS}

The Illumina sequencing datasets determined in this study have been deposited in the BioProject of the BIG Data Center, Beijing Institute of Genomics (BIG), Chinese Academy of Sciences, under the accession number PRJCA003739. The data are publicly accessible at http://bigd.big.ac.cn/gsa.

\section{CONFLICT OF INTEREST}

The authors declare that the research was conducted in the absence of any commercial or financial relationships that could be construed as a potential conflict of interest.

\section{AUTHOR CONTRIBUTIONS}

Q.L., Z.C., and T.X. wrote the manuscript text, demonstrated majority of the bioinformatics analysis and prepared most of the figures and tables. D.H., H.H., C.H., F.Z., H.G., Z.L., and X.G. supplied Bougainvillea RNA for sequencing and experimental verification. L.C. reviewed the manuscript. Z.J. presided over the 
whole project, designed the experiments, reviewed and revised the manuscript. All co-authors have reviewed the manuscript.

\section{FUNDING}

This work was supported by a grant from the Transportation Technology Project of Fujian Province (No. 2018Y002).

\section{References}

1. Abarca-Vargas, R., and Petricevich, V. L. (2018). Bougainvillea genus: a review on phytochemistry, pharmacology, and toxicology. Evid. Based Complement. Alternat. Med. 2018, 9070927. doi:10.1155/2018/9070927.

2. Anders, S., Pyl, P. T., and Huber, W. (2015). HTSeq-a Python framework to work with high-throughput sequencing data. Bioinformatics 31, 166-169. doi:10.1093/bioinformatics/btu638.

3. Arteaga Figueroa, L., Barbosa Navarro, L., Patiño Vera, M., and Petricevich, V. L. (2015). Preliminary studies of the immunomodulator effect of the Bougainvillea xbuttiana extract in a mouse model. Evid. Based Complement. Alternat. Med. 2015, 479412. doi:10.1155/2015/479412.

4. Bolger, A. M., Lohse, M., and Usadel, B. (2014). Trimmomatic: a flexible trimmer for Illumina sequence data. Bioinformatics 30, 2114-2120. doi:10.1093/bioinformatics/btu170.

5. Cheng, C. Y., Krishnakumar, V., Chan, A. P., Thibaud-Nissen, F., Schobel, S., and Town, C. D. (2017). Araport11: a complete reannotation of the Arabidopsis thaliana reference genome. Plant J. 89, 789804. doi:10.1111/tpj.13415.

6. Choudhary, N., Kapoor, H. C., and Lodha, M. L. (2008). Cloning and expression of antiviral/ribosomeinactivating protein from Bougainvillea xbuttiana. Journal of Biosciences 33, 91-101. doi:10.1007/s12038-008-0025-8.

7. Fu, L., Niu, B., Zhu, Z., Wu, S., and Li, W. (2012). CD-HIT: accelerated for clustering the next-generation sequencing data. Bioinformatics 28, 3150-3152. doi:10.1093/bioinformatics/bts565.

8. Ghogar, A., and Jiraungkoorskul, W. (2017). Antifertility effect of Bougainvillea spectabilis or paper flower. Pharmacogn. Rev. 11, 19-22. doi:10.4103/phrev.phrev_44_16.

9. Haas, B. J., Papanicolaou, A., Yassour, M., Grabherr, M., Blood, P. D., Bowden, J. et al. (2013). De novo transcript sequence reconstruction from RNA-seq using the Trinity platform for reference generation and analysis. Nat. Protoc. 8, 1494-1512. doi:10.1038/nprot.2013.084.

10. Hernandez-Martinez, A. R., Estevez, M., Vargas, S., Quintanilla, F., and Rodriguez, R. (2011). New dyesensitized solar cells obtained from extracted bracts of Bougainvillea glabra and spectabilis betalain pigments by different purification processes. Int. J. Mol. Sci. 12, 5565-5576. doi:10.3390/ijms12095565.

11. Heuer, S., Richter, S., Metzger, J. W., Wray, V., Nimtz, M., and Strack, D. (1994). Betacyanins from bracts of Bougainvillea glabra. Phytochemistry 37, 761-767. doi:10.1016/s0031-9422(00)90354-6. 
12. Huang, X., and Madan, A. (1999). CAP3: a DNA sequence assembly program. Genome Res. 9, 868877. doi:10.1101/gr.9.9.868.

13. Kozomara, A., and Griffiths-Jones, S. (2014). miRBase: annotating high confidence microRNAs using deep sequencing data. Nucleic Acids Res. 42, D68-D73. doi:10.1093/nar/gkt1181.

14. Lagesen, K., Hallin, P., Rødland, E. A., Staerfeldt, H. H., Rognes, T., and Ussery, D. W. (2007). RNAmmer: consistent and rapid annotation of ribosomal RNA genes. Nucleic Acids Res. 35, 3100-3108. doi:10.1093/nar/gkm160.

15. Lowe, T. M., and Eddy, S. R. (1997). TRNAscan-SE: a program for improved detection of transfer RNA genes in genomic sequence. Nucleic Acids Res. 25, 955-964. Available at: http://www.ncbi.nlm.nih.gov/pubmed/9023104. doi:10.1093/nar/25.5.955.

16. Moreton, J., Izquierdo, A., and Emes, R. D. (2015). Assembly, assessment, and availability of de novo generated eukaryotic transcriptomes. Front. Genet. 6, 361. doi:10.3389/fgene.2015.00361.

17. Musacchia, F., Basu, S., Petrosino, G., Salvemini, M., and Sanges, R. (2015). Annocript: a flexible pipeline for the annotation of transcriptomes able to identify putative long noncoding RNAs. Bioinformatics 31, 2199-2201. doi:10.1093/bioinformatics/btv106.

18. Qin, Y., Lin, Z., Shi, D., Zhong, M., An, T., Chen, L. et al. (2020). Integrate heterogeneous NGS and TGS data to boost genome-free transcriptome research. bioRxiv, 2020.2005.2027.117796. doi:10.1101/2020.05.27.117796.

19. Saleem, H., Zengin, G., Ahmad, I., Lee, J. T. B., Htar, T. T., Mahomoodally, F. M. et al. (2019). Multidirectional insights into the biochemical and toxicological properties of Bougainvillea glabra (Choisy.) aerial parts: A functional approach for bioactive compounds. J. Pharm. Biomed. Anal. 170, 132-138. doi:10.1016/j.jpba.2019.03.027.

20. Sangthong, S., Suksabye, P., and Thiravetyan, P. (2016). Air-borne xylene degradation by Bougainvillea buttiana and the role of epiphytic bacteria in the degradation. Ecotoxicol. Environ. Saf. 126, 273-280. doi:10.1016/j.ecoenv.2015.12.017.

21. Simão, F. A., Waterhouse, R. M., loannidis, P., Kriventseva, E. V., and Zdobnov, E. M. (2015). BUSCO: assessing genome assembly and annotation completeness with single-copy orthologs. Bioinformatics 31, 3210-3212. doi:10.1093/bioinformatics/btv351.

22. Smith-Unna, R., Boursnell, C., Patro, R., Hibberd, J. M., and Kelly, S. (2016). TransRate: reference-free quality assessment of de novo transcriptome assemblies. Genome Res. 26, 1134-1144. doi:10.1101/gr.196469.115.

23. Tsai, C. H., Su, H. J., Liao, Y. C., and Hung, T. H. (2005). First report of Bougainvillea spectabilis chlorotic vein-banding virus infecting Bougainvillea plants in Taiwan. Plant Dis. 89, 1363. doi:10.1094/PD-89-1363C.

24. Xie, C., Yuan, J., Li, H., Li, M., Zhao, G., Bu, D. et al. (2014). NONCODEv4: exploring the world of long non-coding RNA genes. Nucleic Acids Res. 42, D98-D103. doi:10.1093/nar/gkt1222.

\section{Figures}




\section{a}

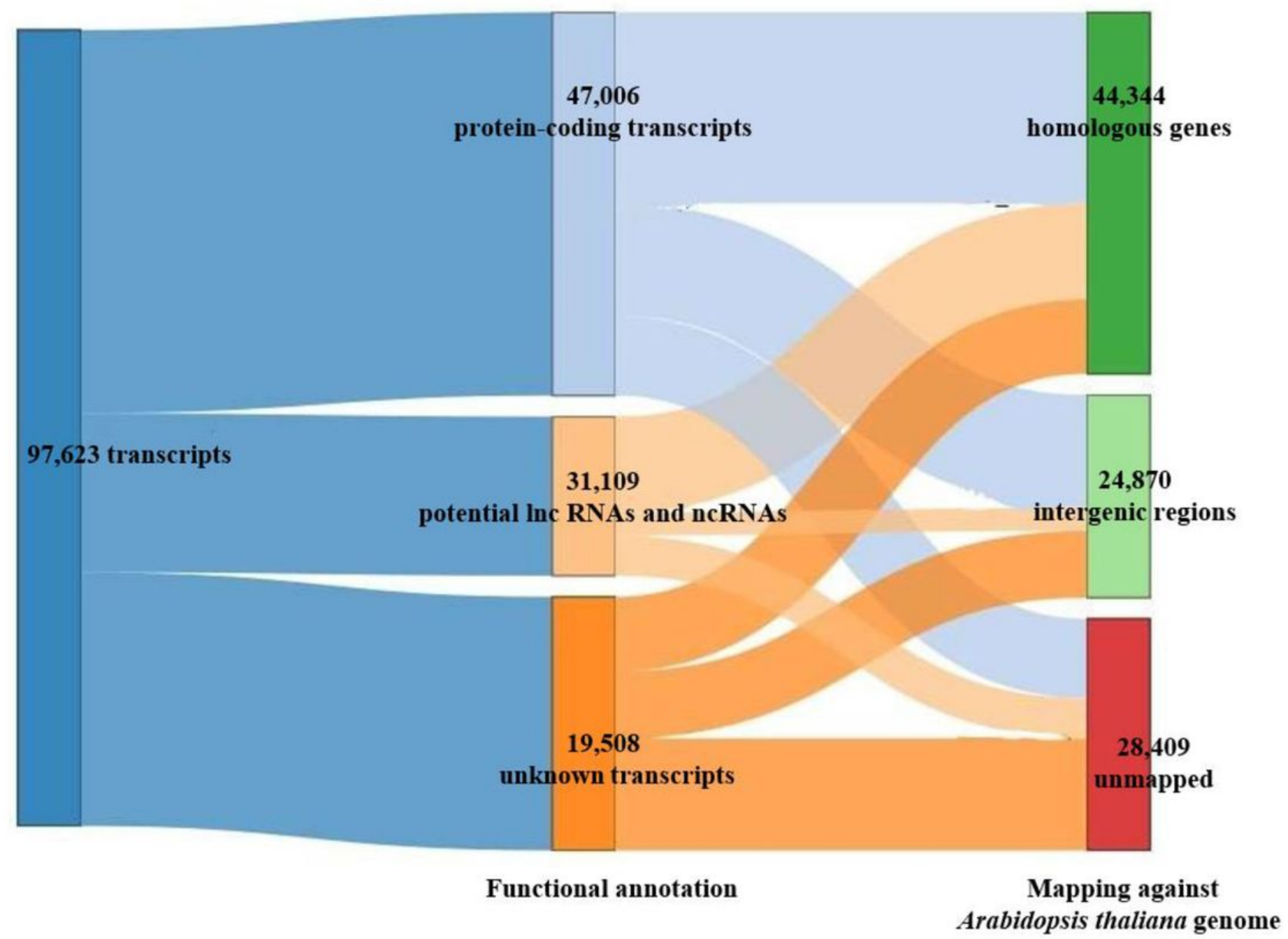

Figure 1

Overview of library transcripts. (a) Functional annotation of library transcripts. (b) Sequence completeness comparison. 
Joint Projects of:

The Bougainvillea is a genus of thorny ornamental vines, bushes, or trees. The inflorescence of Bougainulliea usually consists of three simple waxy flowers, surrounded by large colorful sepal-tike bracts. The abundant color and

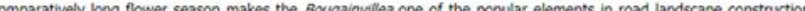
around the world. Unfortunately, up-to-date, no Bougainulilea genome are avallable in public, which largely slows down the pace of mechanistic understanding of Bougainvillea and subsequent applications in practice. Here, we present a sequence library of all expressed transcripts derived from integrating heterogeneous RNA-seq datasets. This transcript library exhibits remarkable capability in reliable molecular and -omics research, serving as an atternative reference to the gnome. It will be particularly useful for systematic and dynamic exploration of gene function under the circumstance of no genome.

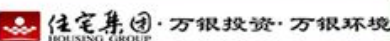

(8) 厦门市公路事业发展虫心

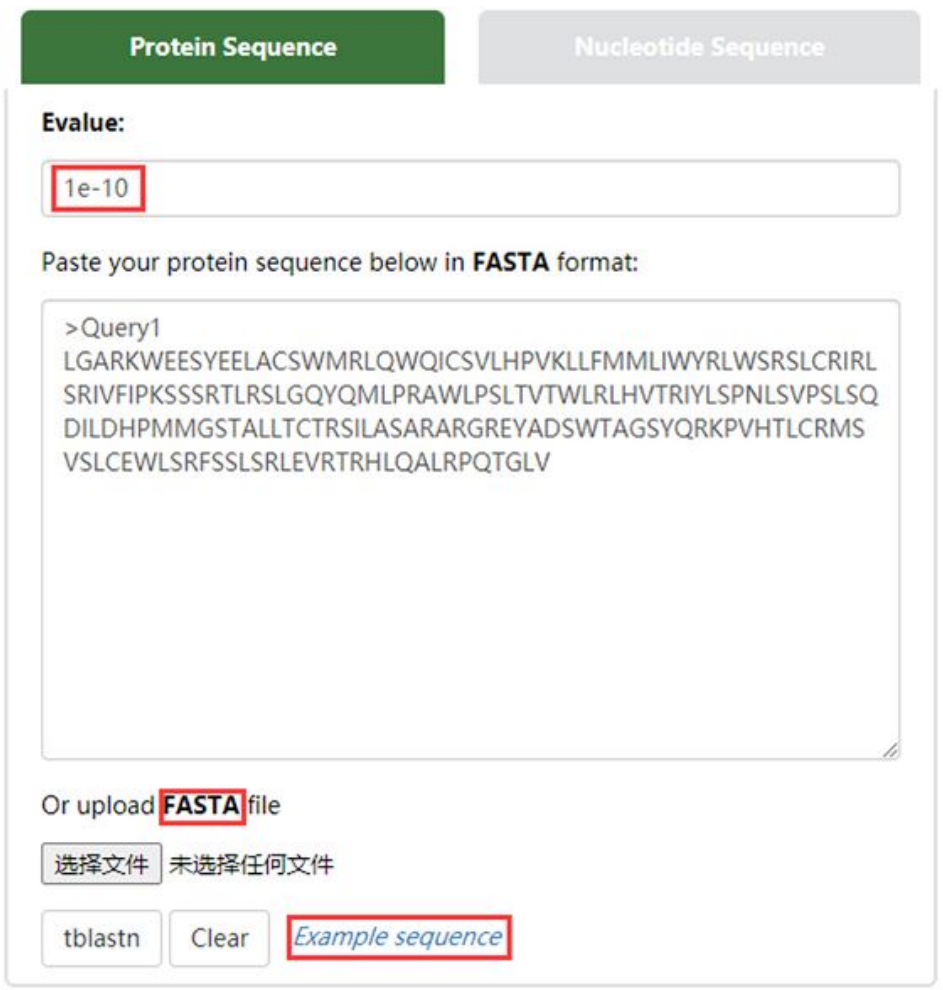

Figure 2

Snapshots of InTransBo user interfaces. (a) Homepage and keyword search form. (b) Blast search form. 
Step1

Step2

Step3 ↔

\section{Figure 3}

You are searching gene with keyword "AP1" Results: 174 item(s)

\begin{tabular}{|c|c|c|c|}
\hline Gene Symbol & Protein Name & Length & \multicolumn{2}{c|}{ Transcript ID } \\
\hline AAP1 & Amino acid permease 1 & 684 & Bt_trans112005_c0_g1_i1 \\
\hline AAP1 & Amino acid permease 1 & 732 & Bt_trans128018_c0_g1_i1 \\
\hline AAP1 & Amino acid permease 1 & 342 & Bt_trans98971_c0_g3_i1 \\
\hline AAP19-1 & AP-1 complex subunit sigma-1 & 492 & Bt_trans113846_C1_g1_i1 \\
\hline
\end{tabular}

$\Downarrow$

\begin{tabular}{|c|c|}
\hline Transcript ID & Bt_trans112005_c0_g1_i1 \\
\hline Gene Symbol & AAP1 \\
\hline Protein Name & Amino acid permease 1 \\
\hline Transcript Length & 684 \\
\hline Transcript Sequence & 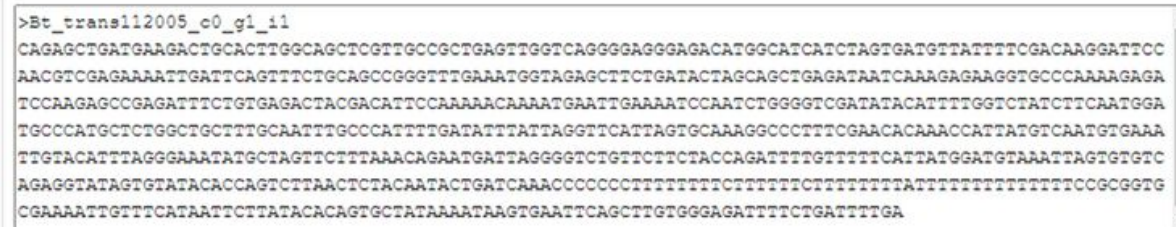 \\
\hline
\end{tabular}

Data retrieval by keyword search method 

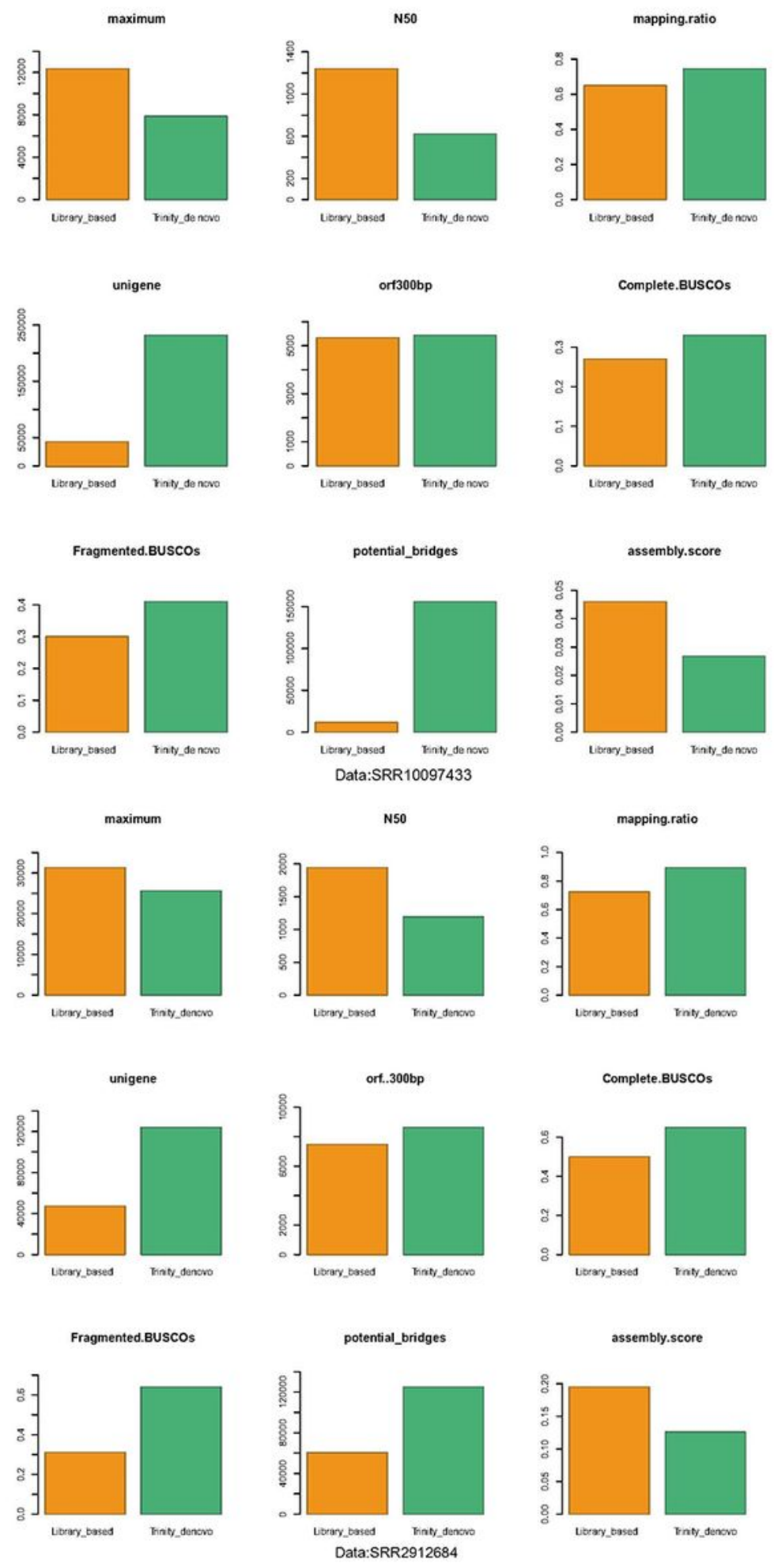

Figure 4

Comparison of transcriptome assembly performance between library-based and de novo Trinity methods 